\title{
The size of knots in polymers
}

\author{
Enzo Orlandini ${ }^{1,2}$, Attilio L. Stella ${ }^{1,2}$, Carlo Vanderzande ${ }^{3,4}$ \\ ${ }^{1}$ Dipartimento di Fisica and CNR-INFM, Università di Padova, I-35131, Padova, \\ Italy \\ ${ }^{2}$ Sezione INFN, Università di Padova, I-35131 Padova, Italy \\ ${ }^{3}$ Departement WNI, Hasselt University, 3590 Diepenbeek, Belgium \\ ${ }^{4}$ Instituut Theoretische Fysica, Katholieke Universiteit Leuven, 3001 Heverlee, \\ Belgium \\ E-mail: carlo.vanderzande@uhasselt.be
}

\begin{abstract}
Circular DNA in viruses and bacteria is often knotted. While mathematically problematic, the determination of knot size is crucial for the study of the physical and biological behaviour of long macromolecules. Here, we review work on the size distribution of these knots under equilibrium conditions. We discuss knot localization in good and poor solvent, or in polymers that are adsorbed on a surface. We also discuss recent evidence that knot size is a crucial quantity in relaxation processes of knotted polymers.
\end{abstract}

PACS numbers: 36.20.Ey, 64.60.Ht, 02.10.Kn, 87.15.Aa 


\section{Knots in DNA}

Knots in DNA of bacteriophages were discovered by J. Wang and coworkers firstly in single-stranded chains [1] and subsequently in double stranded ones [2]. These knots are thought to arise from random cyclization inside the head of the bacteriophage [3]. Subsequent studies of the knotting probability of DNA in vitro and under equilibrium conditions showed a strong dependence on solution conditions $[4,5]$.

On the theoretical side, Delbruck's conjecture [6] that very long ring polymers are almost surely knotted, was proved at about the same time for polygons of $N$ sides $[7,8]$. Simulations of polygons that included the effects of solution conditions, and independently of a 'cylinder model' of DNA, were able to reproduce accurately the experimental knotting probabilities $[9,5]$.

Cells of every type are known to contain topoisomerases (topo's for short), enzymes that can change the topology of DNA. They are mostly involved in regulating the degree of supercoiling, but are also able to knot or unknot DNA rings [10]. Topo's of type I transiently cleave one strand of the DNA [10,11]. When topo I is added to a solution of single stranded DNA (or nicked double stranded DNA), their reaction will lead to a mixture of DNA chains of various topologies. Different knot types are found to occur with frequencies that are determined by equilibrium parameters (temperature, $\mathrm{pH}, \ldots$... Topo's of type II on the other hand carry out reactions on double stranded DNA, require ATP and work under non-equilibrium conditions. Experiments have shown that they lower the knot probability to values far below those found in equilibrium [12]. The precise mechanism behind this simplification of topology remains a matter of debate [13], since it is unclear how a topoisomerase that works locally can recognize the topology of a DNA strand, which is a global property.

Given their biological relevance, the properties of knots in polymers have been investigated also theoretically in the past decade (for a review, see [14]). In the present paper, we review the work that has been done on the question of knot size. Here we define the size of the knot as the smallest part of the polymer that determines the knot type of a given closed ring polymer. Is this part localized in a small region of the backbone or does it spread out over the whole polymer? We believe that this question is relevant biologically for various reasons. Firstly, knot localization could be a crucial factor in understanding the working of type II topoisomerases $[15,16,17]$. Secondly, the size of a knot definitely influences dynamical properties such as the speed by which a DNA ring moves through a gel in an electrophoresis experiment. Indeed gel electrophoresis is, besides direct imaging, one of the few methods available to determine knot type [18].

One of the main insights that has emerged from studies of knot sizes is that size distributions can be described in terms of a scaling picture involving universal critical exponents and scaling functions. From a theoretical point of view, this has the advantage that knot localization can be studied in the simplest models within a given universality class. In the remainder of this review, we will therefore mostly discuss results that were obtained from equilibrium studies of self-avoiding polygons on a lattice [19]. Interactions 
such as bending rigidity and screened Coulomb interactions are known not to influence universal quantities for polymers in a good solvent regime.

This review is organised as follows. In section 2, we discuss the case of knots in a polymer in a good solvent. In section 3, we review the effects of short-range self interactions (such as those occurring in a poor solvent) and of interactions with a surface. In section 4, we discuss some recent results on the dynamics (still in equilibrium) of knotted polymers, for which knot size turns out to be a crucial parameter. Finally, in section 5 we present our conclusions and point out some open questions in this field.

\section{The good solvent regime}

\subsection{Background and early work}

The simplest model for a polymer in a good solvent is the self-avoiding walk (SAW) [19], which is a lattice walk that never visits the same vertex twice. Since mathematically knots can only occur in closed curves (or ring polymers) we will here consider only the special subset of SAW for which the last vertex visited is a nearest neighbour of the first one. Then the walk can be closed into a self-avoiding polygon (SAP) by adding one extra edge. Figure 1 shows a SAP with a (trefoil) knot in it (for an elementary introduction to knot theory, see [20]). The number of edges, which equals the number of vertices for a SAP, will be denoted by $N$. This quantity will be referred to as the 'number of monomers', even though lattice models provide a description of polymers on a coarse-grained scale where a step typically corresponds to a length of the order of a persistence length $L_{p}$ [19]. For dsDNA, $L_{p} \approx 50 \mathrm{~nm}$, which is much larger than the size of a monomer.

The radius of gyration of a polymer is defined as the average squared distance between a monomer (at position $\vec{R}$ ) and the centre of mass of the polymer $\vec{R}_{c m}$

$$
R_{N}^{2}=\frac{1}{N} \sum_{i=1}^{N}\left(\vec{R}_{i}-\vec{R}_{c m}\right)^{2}
$$

The number of distinct $N$-step SAPs will be denoted as $q_{N}$. Since this number equals the partition sum of self-interacting polymers in the limit $T \rightarrow \infty$ (see section 3.1 ), we also refer to it as the partition sum. For $N$ large, one expects the following behaviours [19]

$$
\begin{aligned}
& \left\langle R_{N}^{2}\right\rangle \sim N^{2 \nu} \\
& q_{N} \sim \mu^{N} N^{\alpha-2}
\end{aligned}
$$

where $\langle\cdot\rangle$ stands for a configurational average. The exponents $\nu$ and $\alpha$ are connected through the relation $\alpha=-d \nu+2$ where $d$ is the dimension of space. It is known that in $d=2, \nu$ equals $3 / 4$ exactly whereas in $d=3, \nu \approx .588[21,22]$ The constant $\mu$ is called the connective constant and its logarithm equals the free energy of the polymer per monomer in dimensionless units. 


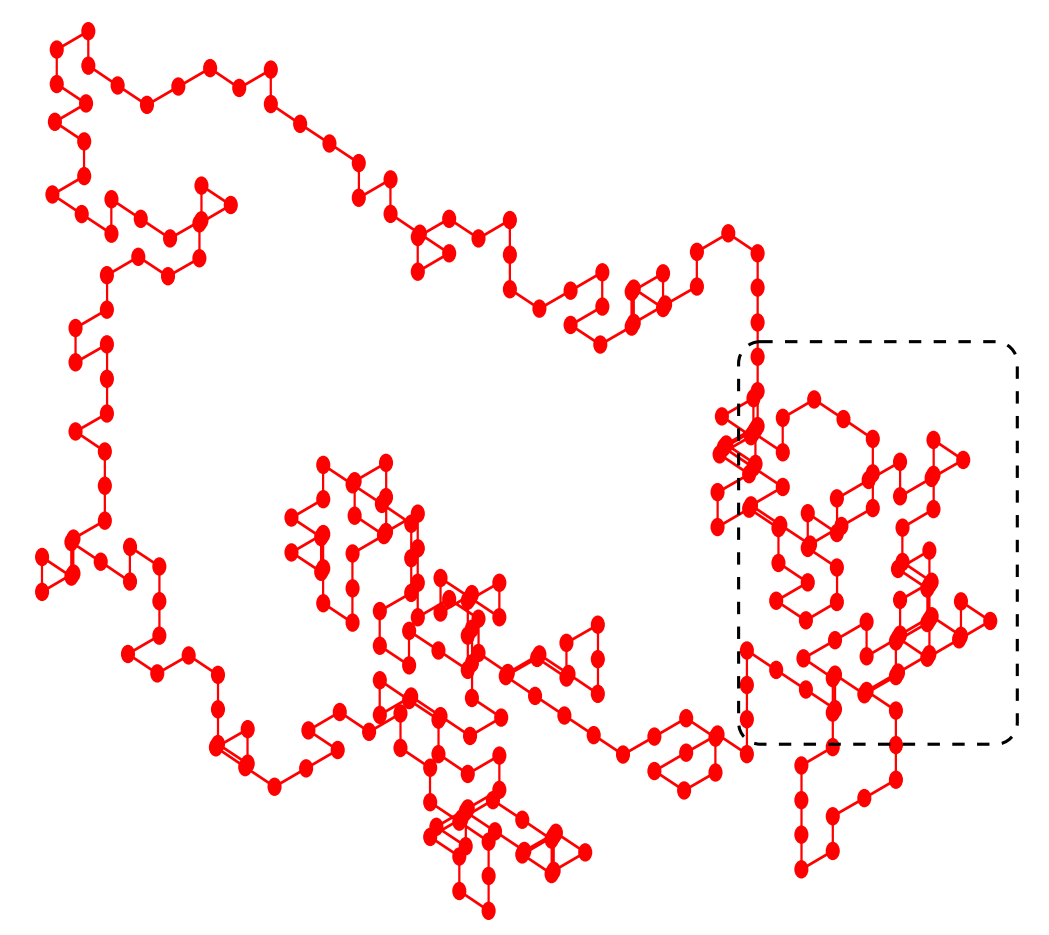

Figure 1. Typical configuration in a good solvent of a self-avoiding polygon (on a cubic lattice) containing a trefoil. Using the method explained in section 2.3, one finds that the knot is localized within the dashed region.

Before beginning the discussion of knot size, we mention that a knot is called a prime knot if it cannot be expressed as a sum of two other, nontrivial knots [20]. The familiar trefoil, or $3_{1}$, knot is an example of a prime knot. In this paper we use the standard notation in which the symbol $n_{l}$ denotes the $l$-th knot with crossing number $n$ (see section 2.2 for a definition of crossing number) in a list that first appeared in [23]. A knot that is not prime is called composite. The composition of two knots $\kappa_{1}$ and $\kappa_{2}$ is denoted by $\kappa_{1} \sharp \kappa_{2}$ and the number of (nontrivial) prime knots appearing in any knot is called its prime factor.

Consider a SAP with a knot $\kappa$ and let $n_{p}$ be its number of prime factors. The first evidence that knots are localized came from numerical studies of the number $q_{N}(\kappa)$ of SAPs of $N$ vertices that contain the knot $\kappa$ [24]. These studies showed that

$$
q_{N}(\kappa) \sim \mu(\kappa)^{N} N^{\alpha(\kappa)-2}
$$

where the entropic exponent is simply given by $\alpha(\kappa)=\alpha(\emptyset)+n_{p}$. Here $\emptyset$ denotes the unknot. This result shows that each prime factor of the knot just multiplies the partition function $q_{N}(\kappa)$ by a factor $N$. This is precisely what one expects if the knot (or prime factor of it) is a localized object that contains $O(1)$ vertices, since such an object can 

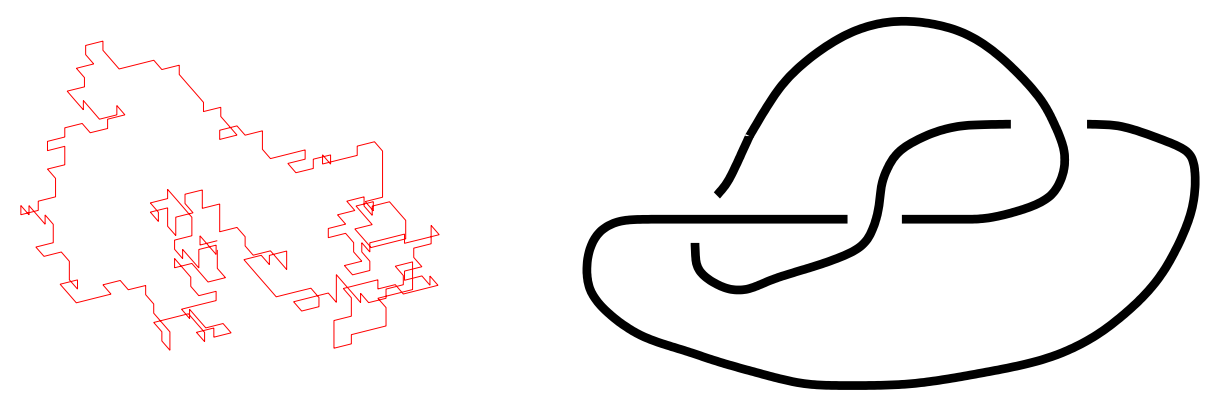

Figure 2. Projection of the polygon in Figure 1 onto a plane (left). Schematic drawing of a projection of a trefoil knot with a minimal number of crossings (right).

be placed at $O(N)$ places on the polymer.

A similar conclusion was drawn from simulations of closed "off-lattice" random walks [25]. These authors investigated the situation of polymers at the $\theta$-point (see section 3.1). To determine knot size, a "window" of $n<N$ monomers of the polymer is considered. This window is moved over the polymer ring. For each location, the window is made into a closed curve by adding an external closure. It is then checked to see whether the resulting construction still contains the original knot. The smallest $n$-value for which this is the case defines the size of the knot. This size was found to be usually rather small (e.g. for a trefoil it typically contains seven segments) and increases with knot complexity. However, no systematic exploration of the dependence of knot size on polymer length was made.

\subsection{Flat knots}

An important breakthrough in the study of knot size was made following the introduction of flat knots [26, 27].

In knot theory, different knots are distinguished by knot invariants, several of which are determined from a projection of the three-dimensional knotted curve onto a plane. Such a projection typically contains a number of over- and undercrossings. The minimal value of this for a given knot type is a topological invariant that is known as the crossing number $n_{c}$. In Figure 2 (left panel), a projection of the trefoil in Figure 1 is drawn, together with a schematic drawing of a trefoil with the minimal crossing number which equals three (Figure 2 right panel). These minimal representations were named flat knots in ref. [27]. Physically, they can be realised by a polymer that is very strongly adsorbed on a surface (for an experimental realisation using DNA, see [28]).

To explain how the size of the flat knot can be defined, consider for example the minimal projection of the trefoil in Figure 2. It consists of six arcs (each going from one 


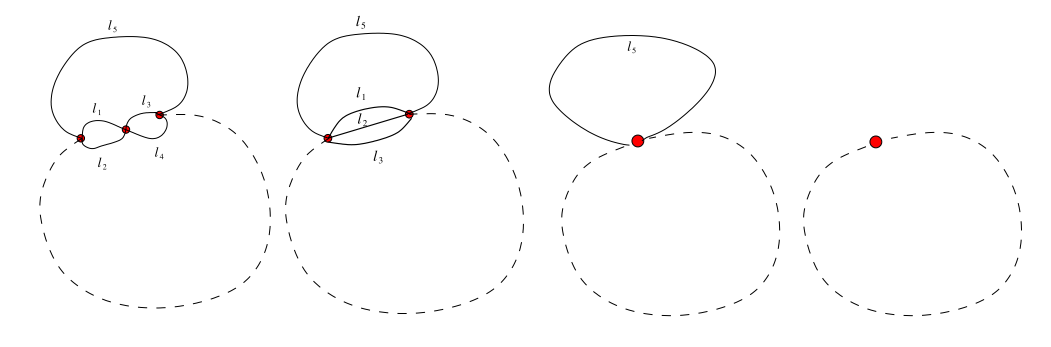

Figure 3. Coarse-grained view of a knotted polymer with (left to right) no, one, four, and five microscopic arcs.

crossing to the next one) of length $l_{6} \geq l_{5} \geq \ldots \geq l_{1}$. Clearly, at least $l_{6} \sim N$. The size of the knot is then defined as $l=N-l_{6}$.

To continue, consider first the case in which four arcs contain only a few monomers (such arcs will be called microscopic), so that the polymer, on a coarse-grained scale, looks like a figure eight (Figure 3 , third panel). In this case the knot length equals $l_{5}$. Following [27], we now present a scaling argument to determine how $l=l_{5}$ scales with $N$. Therefore, the number $q_{N}(l)$ of polymer configurations in the shape of a figure eight has to be determined firstly. This quantity can be obtained from Duplantier's theory of polymer networks $[29,30]$ and is found to be equal to

$$
q_{N}(l)=\mu^{N}(N-l)^{\gamma_{1}-1} F\left(\frac{l}{N-l}\right)
$$

where $\gamma_{1}$ is an exponent that can be determined from conformal field theory and $F$ is a scaling function. When $l \ll N$, we expect that the behaviour of (5) should crossover into that of (3). This fixes the small $l$ behaviour of $F$ and finally leads to the prediction

$$
q_{N}(l) \approx \mu^{N} N^{\alpha-2} l^{-c} \quad l \ll N
$$

where $c=-\left(\gamma_{1}+1+\alpha\right)$. Inserting the known exponent values [29] gives $c=43 / 16$. From (6), one finds that the average length of the knot scales as $\langle l\rangle \sim N^{t}$ where $t=\max (0,2-c)$ (When $c<1$, a cutoff at large $l$ leads to $t=1$ ). When $t=0$, we say that the knot is strongly localized, while for $0<t<1$ we say that it is weakly localized. Finally, the situation with $t=1$ represents a delocalized knot. For knots in the shape of a figure eight, we thus conclude that $t=0$ and that the knot is strongly localized. This means that $l_{5}$ in Figure 3, third panel, does not increase as the total length $N$ of the polymer increases. One can subsequently do a similar analysis for other coarse-grained realisations (also called contractions) of the flat knot. As an example, we show in Figure 3 examples of a polymer with one or no microscopic arc. The authors of [27] analyzed these and all other possibilities using polymer network theory and found that for all cases the knot is strongly localized. Therefore, on a coarse grained scale, the knot is just like a dot on the polymer (Figure 3, rightmost panel). 


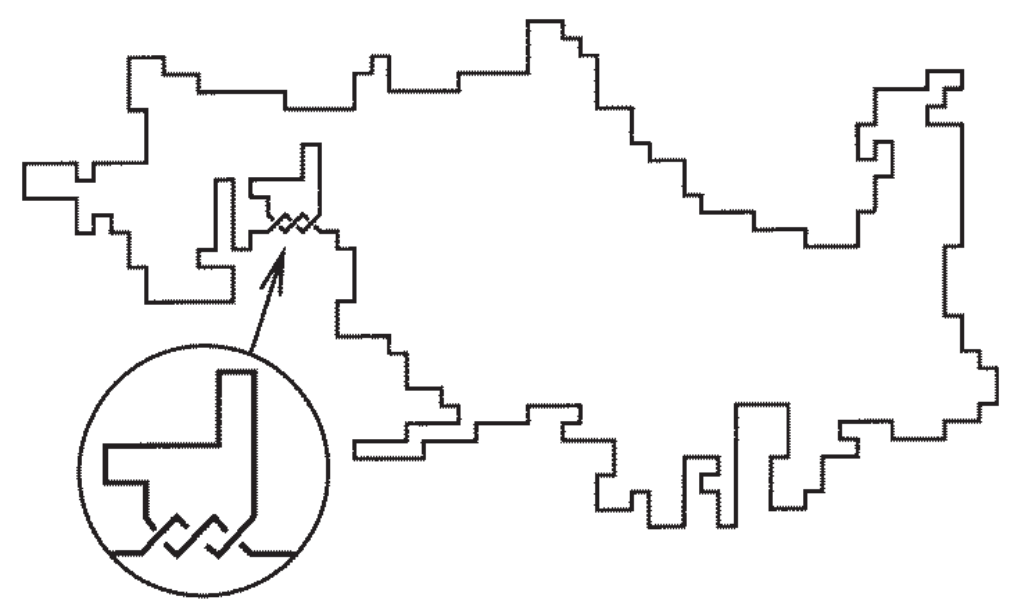

Figure 4. Typical configuration of a flat (trefoil) knot as simulated using the model of [26] for $N=312$. The inset shows the knotted region in more detail.

The results (5) and (6) clearly show that the size of the knot in a polymer is a fluctuating quantity, whose probability distribution can be described in terms of a scaling picture.

Numerical studies of flat knots are in complete agreement with the field theoretic predictions [27]. An elegant lattice model to study flat knots was introduced in [26]. This model starts from SAPs on a two-dimensional lattice, but also includes crossings that represent those occurring in a knot projection. When the fugacity for crossings is very small, the model adequately describes flat knots. Figure 4 shows a typical configuration for a trefoil in this model and as can be seen the knot is strongly localized.

\subsection{The three dimensional case}

While the analytical determination of the exponent $c$ (or $t$ ) is not possible in three dimensions, the scaling picture presented in the previous subsection should still be valid. In [31, 32], knot size in three-dimensional SAPs was studied using two numerical approaches. The first one was an improvement of "the moving window" method, discussed in section 2.1. In contrast with the work of [25], the closure is not chosen randomly but in a deterministic way such as to avoid as much as possible intersecting the pre-existing skein [20]. In this way, systematic errors that can occur when window closure changes the topology are minimized. Figure 5 shows some results from this approach. Plotted is the average knot length versus the number of monomers for rings with a trefoil knot. The data are clearly consistent with a power law behaviour $\langle l\rangle \sim N^{t}$ and give the exponent estimate $t \approx .75$.

More precise estimates for this exponent can be found from analyzing higher 


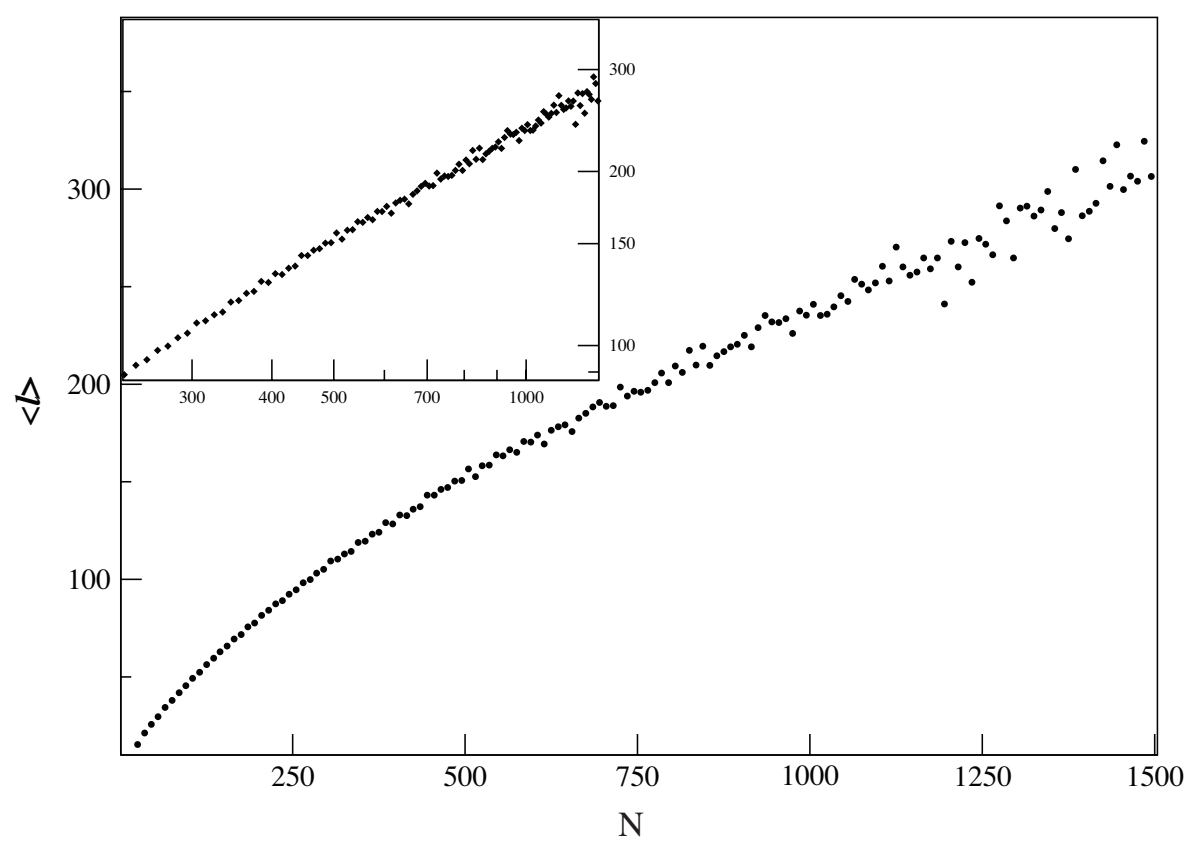

Figure 5. Average length of a trefoil knot as a function of $N$. The inset shows the same data on a log-log plot.

moments of the length distribution. Indeed, from the scalings (5) and (6) it follows that the probability $p(l, N)$ to find a knot of length $l$ in a ring of $N$ monomers has the form

$$
p(l, N)=l^{-c} f\left(l / N^{D}\right)
$$

Here the scaling function $f$ approaches zero very rapidly for $l>N^{D}$. For flat knots, $D=1$ and this is also what can be expected in three dimensions, since we do not expect that there exists an extra topological length scale $N^{D}$ in a polymer. From (7), it follows that the $q$-th moment of the length distribution, $\left\langle l^{q}\right\rangle$, grows with $N$ with an exponent $t(q)=D q+D(1-c)$. Hence, by calculating $\left\langle l^{q}\right\rangle$ from the simulation data for a range of $q$-values, estimates of $c$ and $D$ can be obtained. The results for various knot types are given in table 1 . Notice that $D$ is reasonably close to the value that was expected, especially for the $3_{1}$ case. For more complex knots, polygons of larger $N$ need to be simulated. Since the statistics becomes poorer with increasing $N$, we can understand why the discrepancy between the expected value of $D$ and the measured one increases for more complicated knots. For the same reason, it is impossible to conclude from these data whether or not $t$ depends on knot type. However, these data clearly show that knots in a good solvent are weakly localized.

Independent confirmation from this comes from a second approach to knot size in 
Table 1. Estimates of the exponents $c$ and $D$ for various knot types as determined from the moments of the length distribution (good solvent regime).

\begin{tabular}{cccc}
\hline Knot type & $D$ & $c$ & $t$ \\
\hline $3_{1}$ & $0.958 \pm 0.004$ & $1.25 \pm 0.04$ & $0.72 \pm 0.03$ \\
$4_{1}$ & $0.934 \pm 0.004$ & $1.18 \pm 0.04$ & $0.77 \pm 0.03$ \\
$5_{1}$ & $0.918 \pm 0.002$ & $1.14 \pm 0.03$ & $0.79 \pm 0.03$ \\
$5_{2}$ & $0.863 \pm 0.003$ & $1.11 \pm 0.04$ & $0.76 \pm 0.04$ \\
$7_{1}$ & $0.864 \pm 0.004$ & $1.06 \pm 0.09$ & $0.81 \pm 0.09$ \\
\hline
\end{tabular}

which a SAP is partitioned into two mutually avoiding loops which each contain a knot. We denote the knot types by $\kappa_{1}$ and $\kappa_{2}$ (each of which could be the unknot). The two parts of the polygon are separated by a slip link that does not allow a complete migration of one knot into the other loop (Figure 6). Only cases in which the two loops are not linked were investigated. Consider first the case for which $\kappa_{1}=\kappa_{2}$. Since the number of configurations is much greater when one of the loops is much larger than the other one, most configurations show a marked length asymmetry in which the larger loop contains $O(N)$ monomers while the smaller one typically contains just enough length to store its knot. A similar result is found when $\kappa_{1} \neq \kappa_{2}$. In this case, for $N$ not too large, the smaller loop is generically the one containing the 'simpler' knot. The length of the smaller loop is again found to be a fluctuating quantity (unless $\kappa_{1}$ is the unknot) and it can be argued that this length has the same scaling behaviour as the size of the knot it contains [32]. In this way, independent estimates of the exponents $t$ and $D$ can be obtained which are in good agreement with those obtained from the direct measurements discussed previously.

In conclusion, the available evidence shows that in a good solvent, knots are weakly localized with an exponent $t \approx .75$.

\section{Collapse and adsorption}

\subsection{Collapse}

Excluded volume forces that swell a polymer become weaker when the temperature is lowered. It is well known [19] that these effects lead to the collapse of a polymer below the $\theta$-temperature, $T_{\theta}$. One may then wonder what happens to knot size at and below $T_{\theta}$. This could give a first indication of what can be expected for knots in the native state of a protein. This question was investigated both for flat knots [33, 34] and for the three-dimensional case [31, 32] using the approaches discussed in the previous section.

The case of flat knots was studied by adding attractive self-interactions to the model of reference [26]. As usual [19], this is done by assigning an attractive energy with each pair of nearest neighbor lattice vertices that is visited by nonconsecutive edges. The $\theta$ point of the resulting model is located at $1 / T_{\theta}=0.67 \pm .02$. In Figure 7 , numerical results for a polymer with a trefoil are plotted. Shown are data for $\left\langle l_{5}\right\rangle$ at various temperatures. 


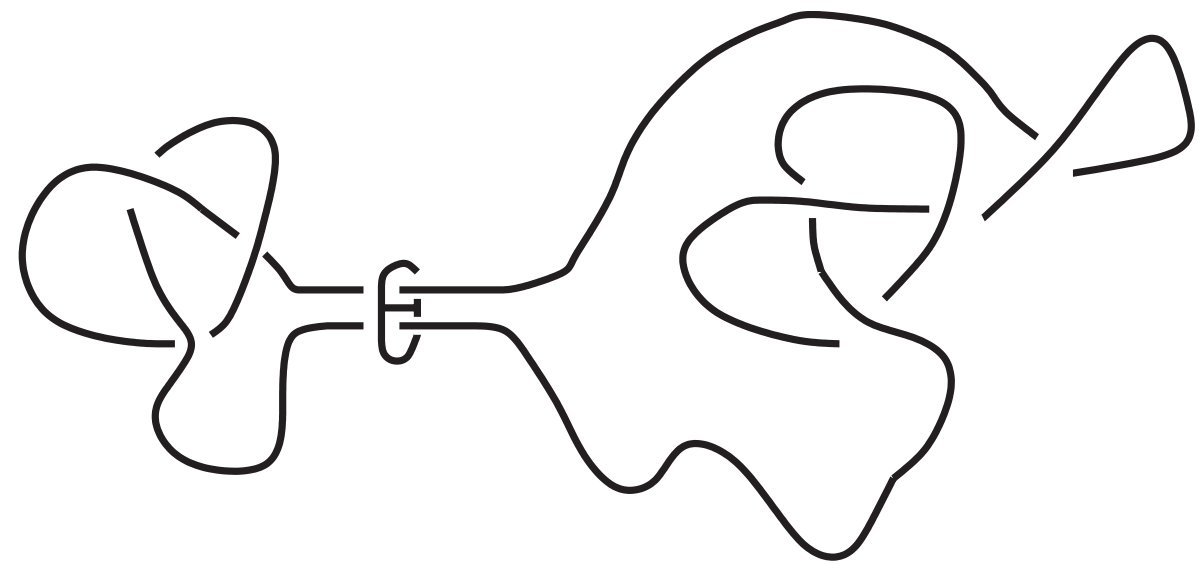

Figure 6. A loop consisting of two mutually avoiding loops separated by a slip link. Each of the two parts can contain a knot that cannot migrate completely through the slip link.

At high temperatures (lower curves) one recovers the knot localization found earlier in the good solvent regime. But this behaviour changes at lower temperatures. Precisely at the $\theta$ temperature, the knot becomes weakly localized with an exponent $t=.44 \pm .02$. The length of the four smaller arcs remains very small so that on a coarse grained level the knotted polymer looks like a figure eight. Finally, at temperatures below $T_{\theta}$, the polymer collapses and the knot delocalises $(t \approx 1)$. Moreover, the average length of smaller arcs such as $\left\langle l_{4}\right\rangle$ also starts to grow proportional to $L$. Here the simple figure eight picture breaks down.

In Figure 8 we show a typical configuration of a knotted polymer below the $\theta$-point. The crossings are spread over the graph and this is in sharp contrast with the situation shown in Figure 4.

The picture presented here is confirmed by an analysis of the distribution of knot lengths. At $T_{\theta}$ the distribution is power law with an exponent $c=-1.63$, while in the collapsed phase, the distribution is power law only for small $l$, while it flattens for larger knot sizes [33]. Completely similar results where found for polymers containing other types of prime knots such as the $5_{1}$ or $7_{1}$ and for the composite knot $3_{1} \sharp 3_{1}$ [35].

As was the case for flat knots in a good solvent, one can obtain an exact expression for the exponent $c$ (and hence $t$ ) from network theory. For polymers at the $\theta$-point, this theory was established by Duplantier and Saleur [36]. Application to the figure eight network that interests us here gives $c=11 / 7 \approx 1.57$ and $t=3 / 7 \approx .43$ in very good agreement with the numerical estimates quoted above. However, an analysis of other contractions of the trefoil knot shows that the configuration shown on the left of Figure 3 has the smallest $c$-value $(c=5 / 7$ and thus $t=1)$ and should dominate 


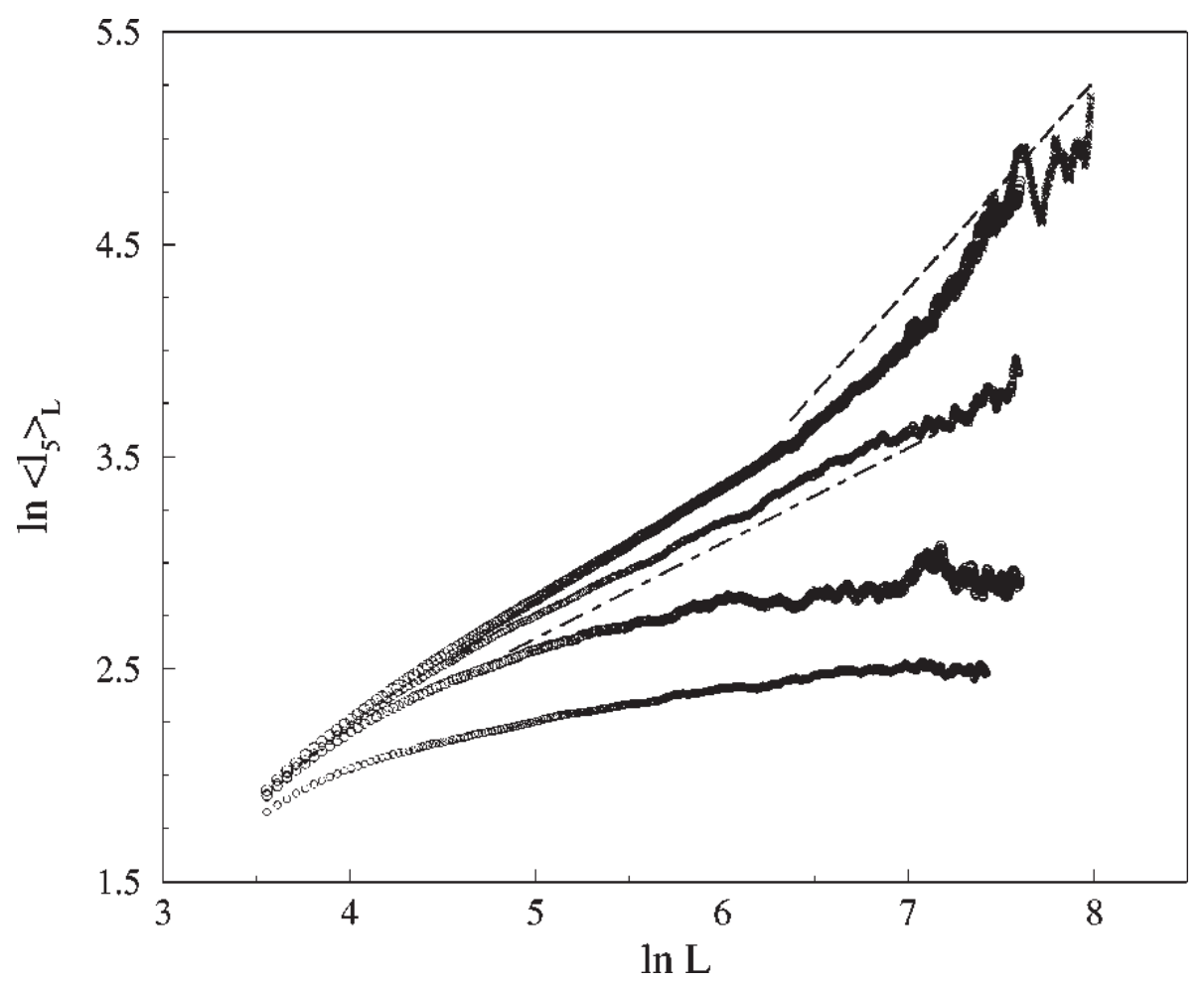

Figure 7. Log-log plot of $\left\langle l_{5}\right\rangle$ as a function of the number of monomers (denoted here by $L$ ) for a polymer with a trefoil (for flat knots with self-interactions). From bottom to top: $1 / T=0.0,0.5,0.67$ and 0.8 . The dot-dashed line has slope 0.44 while the dashed one has slope 1.0 .

polymer configurations with a trefoil [34]. This theoretical analysis, which predicts a delocalization even at the $\theta$-temperature of flat knots is in contradiction with the numerical results as discussed above. The reason for this discrepancy between theory and simulation is at this moment unclear. There is however no doubt that below $T_{\theta}$ flat knots delocalise.

The moving window method to determine knot size in three dimensions fails in the collapsed phase. The reason is simple: because of the compact nature of the polymer, the topology of the knotted part is changed with high probability after closure of the window. Fortunately, the method based on entropic competition can still be used even though in the dense phase Monte Carlo simulations become considerably slower. Good estimates of the exponents $c$ and $D$ can be obtained from analysis of various moments of the knot size distribution (see section 2.2). As an example, in Figure 9 the expont $t(q)$ is plotted for a polymer with a trefoil. From these data one estimates $c \simeq 1.1$ and $D \simeq 0.98$. Similar results are found for the figure eight $\left(4_{1}\right)$ knot. Together these data imply that also in $d=3$ knots are delocalized in the collapsed phase. Independent evidence for this 


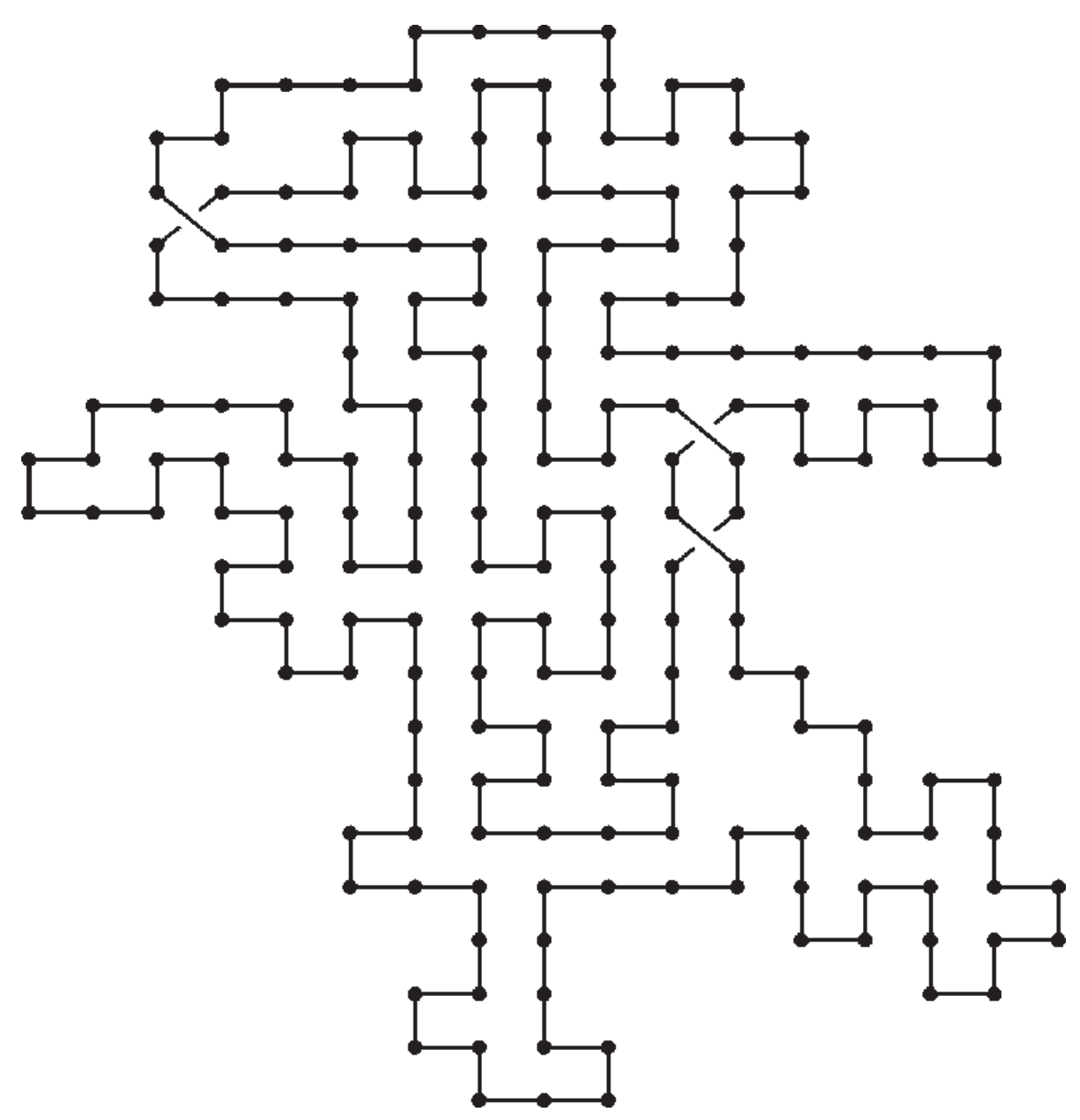

Figure 8. Typical configuration of a (flat) polymer with a trefoil knot in the low $T$ collapsed phase.

behaviour was also found in an off-lattice model of (knotted) polyethylene [37].

\subsection{Adsorption}

Polymers can get adsorbed onto a surface when there is an attractive interaction between the monomers and the surface. If the surface is a large impenetrable flat wall, the polymer will undergo a well known adsorption transition [19,38]. At high temperatures, only a finite number (in the limit $N \rightarrow \infty$ ) of monomers is on the surface. Below the adsorption transition at temperature $T_{a}$ a finite fraction (i.e. proportional to $N$ ) of the monomers becomes adsorbed. This fraction increases with decreasing temperature until at zero temperature the full polymer gets adsorbed. In the case of a knotted ring however, some monomers fail to adsorb because topology requires the adsorbed polymer 


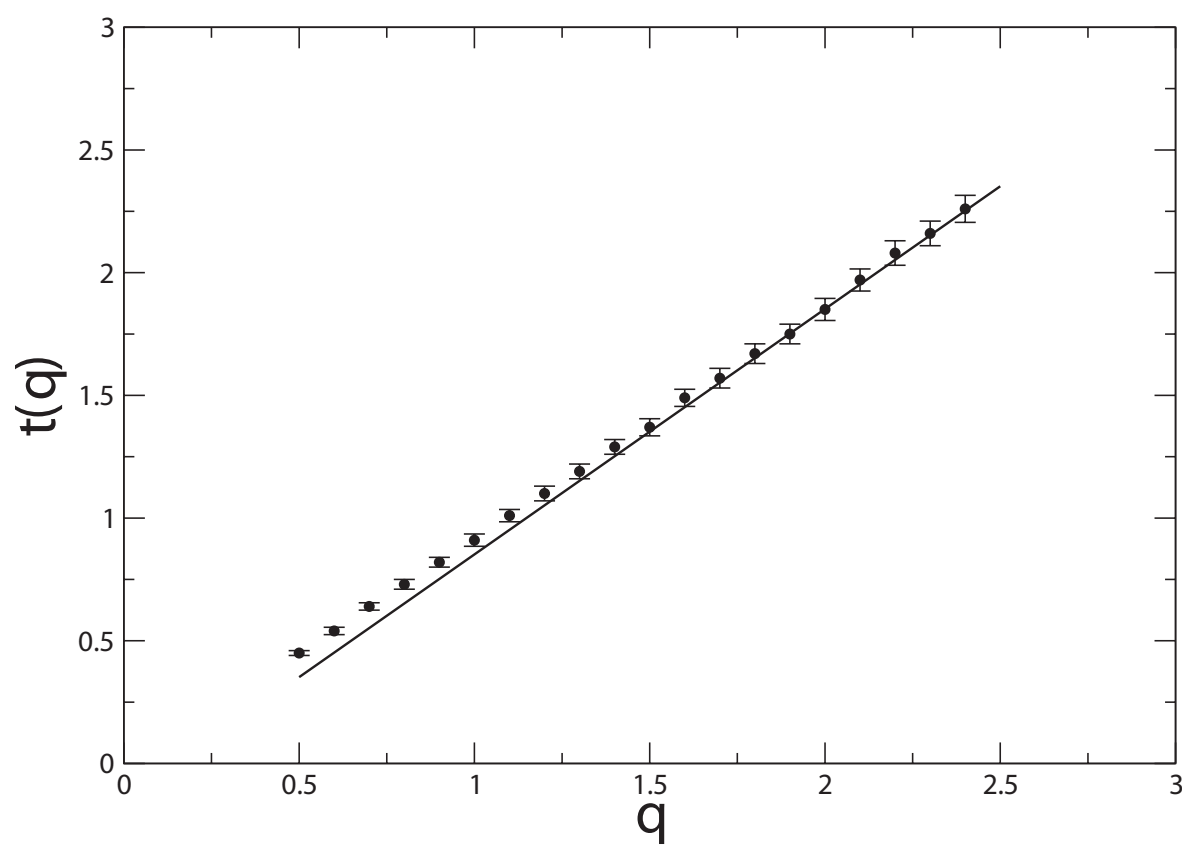

Figure 9. Exponent $t(q)$ in the compact phase as determined from the entropic competition technique (with two trefoils).

to have a minimal number of crossings. One therefore expects that the fully adsorbed polymer assumes precisely the shape of a flat knot. Thus in adsorption phenomena, it is possible to see how a knotted polymer (e.g. in a good solvent) crosses over from the weak localization typical of three dimensions (at high $T$ ) to the strong localization of flat knots at zero temperature. In a recent study this interplay between topology and adsorption was elaborated using a standard lattice model for polymer adsorption [39]. In this model, the polymer is restricted to a half space (say $z \geq 0$ ) and has an energy $-N_{a}$, where $N_{a}$ is the number of monomers in the surface $z=0$. Numerical calculations have determined the adsorption transition accurately at $T_{a} \approx 3.497$ [40]. The study of [39] could be relevant to understanding experiments that indicate that the $\nu$-exponent of knotted and adsorbed DNA can have a value intermediate between the three-dimensional and the two-dimensional one [41], even though in this experiment the polymer may be adsorbed in non equilibrium configurations.

We now discuss the results of [39]. In this work, knot size was determined using the moving window method. Figure 10 shows some typical results, again for a trefoil knot. The upper curve indicates that even at a temperature $T=3.5$, which is just above the adsorption transition, the weak localization typical of the three-dimensional situation is recovered. The curve almost coincides with that of the non interacting case $(T \rightarrow \infty)$, which is shown as a dashed curve. The same holds at all temperatures above $T_{a}$. 
Table 2. Estimates of the exponent $c$ for different values of $T$ in the adsorption model and for trefoil knots. These values were obtained from a finite size analysis of the moments of the knot length.

\begin{tabular}{cc}
\hline$T$ & $c$ \\
\hline 3.50 & $1.27 \pm .03$ \\
2.75 & $1.55 \pm .05$ \\
2.00 & $1.80 \pm .05$ \\
1.50 & $2.42 \pm .10$ \\
1.25 & $2.55 \pm .10$ \\
\hline
\end{tabular}

Below the adsorption transition the situation changes significantly: the average knot length still follows a power law behaviour as a function of $N$, but the exponent $t$ decreases with $T$. At still lower temperatures, $\langle l\rangle$ goes to a constant for large $N$. This is a signal of the strong localization regime as found for the case of flat knots. To investigate this issue further, one has to determine the $c$-exponent, which as discussed in section 2.2 can be obtained from the moments of the length distribution. The resulting values are given in table 2 . These data show that $c$ increases as the temperature is lowered and tends to the flat knot value of $43 / 16 \simeq 2.63$ at the lowest temperature that could be simulated. From the data it is unclear whether the flat knot value is reached only for $T \rightarrow 0$ or is valid in a certain range of (low) temperatures. The results also convincingly show that the knot strongly localizes at a temperature considerably below $T_{a}$. This, together with the continuous variation of $c$ with $T$, is a rather surprising result. Results for other prime knots are quite similar to those presented for the trefoil knot.

\section{Dynamics of knotted polymers}

So far, we have focused on static properties of knotted polymers. Less work has been done on dynamic behaviour. It has been established that in a good solvent, the presence of a knot doesn't influence the scaling with $N$ of the diffusive behaviour of a polymer [42]. The diffusion constant, and hence the mobility, does however depend on details of the knot topology, as gel electrophoresis experiments have clearly shown $[18,43]$. Here we will review recent work [44] on the time dependence of the knot length autocorrelation and on the diffusion of the knotted region in space. A new time scale $\tau_{t}$ is found to determine these quantities.

In Rouse dynamics [45, 46], a polymer is modeled as a set of beads that are connected by harmonic springs and that are subjected to random thermal forces exerted by the solvent. In a good solvent, one also includes self-avoidance effects. The motion of the monomers is then determined numerically. It is well established that the center of mass of the polymer diffuses with a diffusion constant $D_{c m}$ that is inversely proportional 


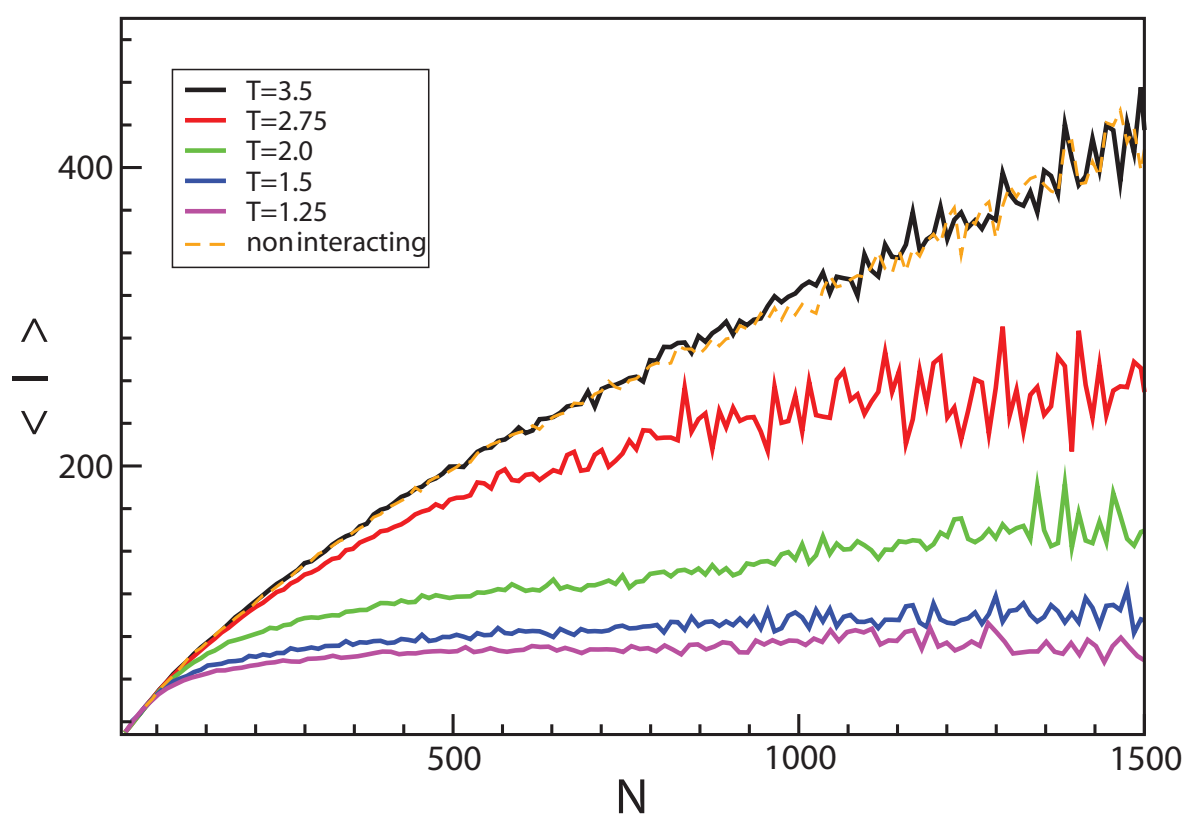

Figure 10. (Color online) Average knot length $\langle l\rangle$ as a function of $N$ for SAPs with a trefoil knot (adsorption model). Different curves correspond to decreasing (from top to bottom) values of the temperature. The dashed curve corresponds to the case without interaction $(T \rightarrow \infty)$.

to the number of monomers $N$. The autocorrelation function of the radius of gyration

$$
\left\langle R_{N}(t) R_{N}(0)\right\rangle_{c} \equiv \frac{\left\langle R_{N}(t) R_{N}(0)\right\rangle-\left\langle R_{N}(t)\right\rangle^{2}}{\left\langle R_{N}^{2}(t)\right\rangle-\left\langle R_{N}(t)\right\rangle^{2}}
$$

is known to decay exponentially with the Rouse time scale $\tau_{R} \sim N^{2 \nu+1}$ (in this section $t$ stands for time). For the three dimensional case, this becomes $\tau_{R} \sim N^{2.2}$. The Rouse time scale corresponds to the time that the polymer needs to diffuse over a distance equal to its radius of gyration. Rouse dynamics neglects some physical effects [46], such as hydrodynamic interactions, but is known to give a good description of several experimental situations, some of which involve DNA (see e.g. [28, 47]).

In the simulations of [44], a lattice model of a knotted ring polymer is investigated. In order to simulate Rouse dynamics, two-bond bead motion (Verdier-Stockmayer) and 3-bond crankshaft motion are combined. It has been shown that with such an approach, universal properties of Rouse dynamics can be simulated correctly [48]. During the simulation, observables such as the centre of mass of the polymer, the center of mass of the knot and the size of the knot are monitored. To determine these last two quantities the moving window technique of [31] is used.

From the data on the knot length as a function of time an autocorrelation function 
can be determined. In Figure 11, the top line shows results for this quantity for $N=400$ for a ring polymer with a trefoil. The decay is exponential with a decay time $\tau_{t}$. From the data for several $N$ values, one finds $\tau_{t} \sim N^{z_{t}}$ with $z_{t}=2.33 \pm .08$. Within the errors, this exponent value is higher than that for $\tau_{R}$. Further evidence that this presents a new time scale comes from an investigation of the autocorrelation function of the radius of gyration. This is also shown in Figure 11 (lower curve). Clearly its decay is doubly exponential. In comparison, for an unknotted ring a pure exponential decay is observed. A further analysis shows that the time constant of the unknot, together with the fast one of the knotted polymer, is proportional to $\tau_{R}$ (this gives an independent verification that the simulation procedure used gives a correct description of Rouse dynamics for unknotted rings). On the other hand, as can be seen in Figure 11, the late time decay of the radius of gyration autocorrelation has the same time constant as that of the length autocorrelation. A quantitative analysis of its $N$-dependence shows that it grows as $N^{2.31 \pm .08}$ supporting this conclusion. Together these data, and those for other type of knots, provide strong evidence for the presence of a new, slow time scale $\tau_{t} \sim N^{z_{t}=2.32 \pm 0.1}$ associated to the time over which the knot length decorrelates.

Another quantity of interest is the diffusion of the knotted region. Firstly, the

monomers that form the knot are located and their centre of mass, $\vec{R}_{c m, k}(t)$, is determined. The diffusion of the knotted region is then given by the function

$$
g_{3, k}(t, N)=\left\langle\left(\vec{R}_{c m, k}(t)-\vec{R}_{c m, k}(0)\right)^{2}\right\rangle
$$

It is found [44] that $g_{3, k}$ has three time regimes. In an early and intermediate region, $g_{3, k}$ increases as a power of time. In the intermediate regime the power equals $\gamma \approx .6 \pm .03$. After a very long time this behaviour crosses over to that of the whole polymer $g_{3, k} \sim t / N$ as it should be since the knot has to follow the motion of the whole polymer eventually. The crossover time is found to scale proportional to $\tau_{t}$ so that this new, slow, topological time scale also plays an important role in diffusive phenomena of knotted polymers.

A simple interpretation of $\tau_{t}$ can be found by assuming that the diffusion of the knotted region along the polymer backbone is normal and that the associated diffusion constant is inversely proportional to knot length, i.e. $D_{K} \sim N^{-t}$ (where $t$ is again the exponent introduced in section 2). A similar assumption was made in another context in [49]. Then the time the knot takes to diffuse along the backbone over the typical length of the knot is proportional to $N^{2 t} / D_{K} \sim N^{3 t}$. The estimates of $t$ given in section 2 then lead to a power $3 t=2.25 \pm .15$, which within the numerical accuracy equals $z_{t}$. If correct, this argument shows that knot length plays a crucial role in determining the dynamical properties of knotted polymers.

\section{Summary and outlook}

In this review, we have seen that thanks to the development of new simulation techniques, the distribution of knot sizes in polymers in equilibrium is now rather well known, both in the physically relevant case of three dimensions and for flat knots, 


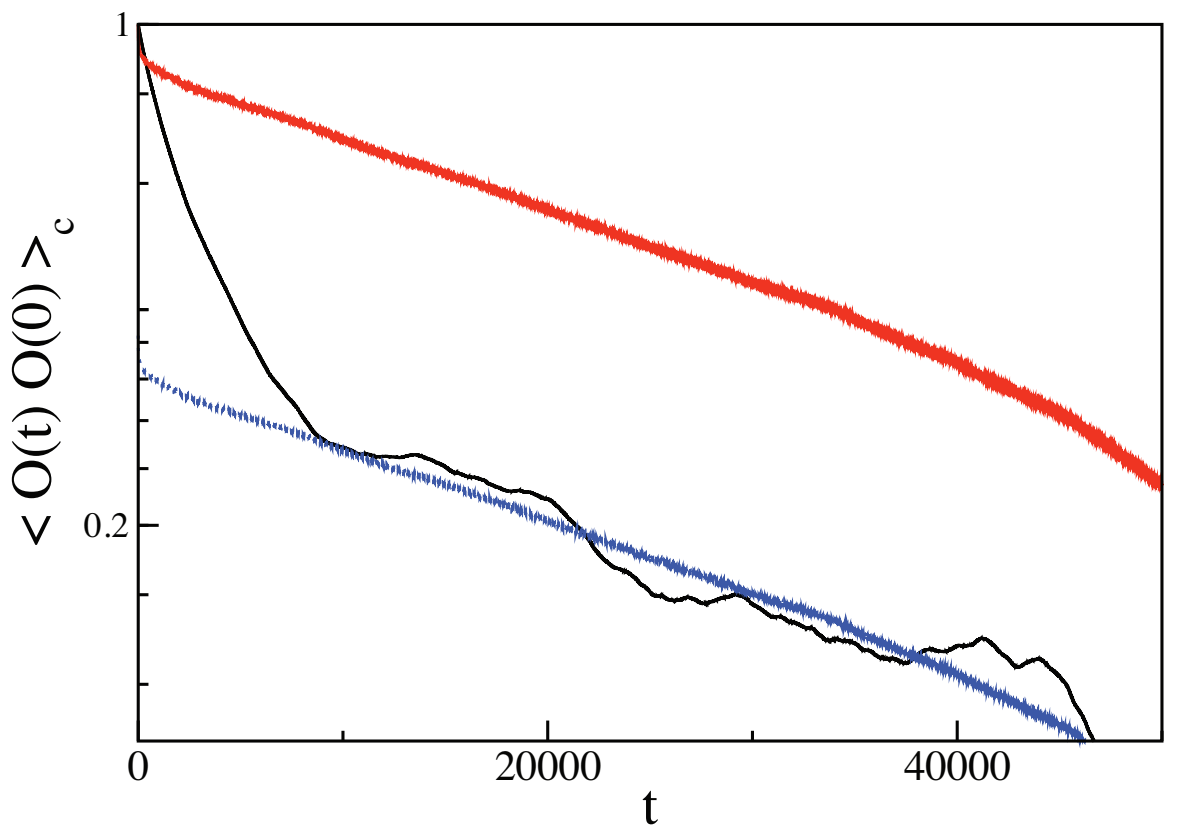

Figure 11. (Color online) Semilogarithmic plot of autocorrelation functions versus time for a SAP with $N=400$. Shown are results for the knot length (upper curve, for which $O(t)=l(t)$ ) and the radius of gyration (lower double exponential curve, for which $\left.O(t)=R_{N}(t)\right)$. The upper curve is also redrawn with a vertical shift downwards in order to show more clearly that both decays have the same late time behaviour.

for which even some analytical insight is possible. In three dimensions, the knot is weakly localized, but it becomes delocalized below the $\theta$-temperature. On the other hand, if the polymer is attached to a surface, the knot will become strongly localized at a temperature below the adsorption one. The knot size distribution exponent $c$ (or $t$ ) could also be relevant to understand length-length autocorrelations and other dynamical quantities such as the diffusion of a knotted polymer or the autocorrelation of its radius of gyration.

The open frontier in this field of research now lies in problems outside equilibrium.

Firstly, we mention the mobility of knotted polymers. It is known that gel electrophoresis separates DNA according to knot type [50]: the more complex the knot, the higher its mobility. These experimental findings can be understood if polymers with more complex knots are more compact and hence have a smaller friction coefficient. A more detailed investigation [18] seems to justify this point of view. It should be remarked 
here that many electrophoresis studies are done on rather small chains, and are therefore outside the scaling regime we focus on in this paper. The precise role, if any, that knot length plays in determining the mobility of a polymer remains to be investigated. It could be that other quantities, such as the smallest sphere enclosing the entire polymer [51] are more relevant. As an interesting related issue, we point out that it has been argued that the gel forces a ring polymer to assume a branched shape [52]. We are currently investigating knot localization in a ring polymer in an array of obstacles [53] as a first step in elucidating the possible role of knot length in the electrophoresis of ring polymers.

Secondly, as mentioned in the introduction, topo's of type II are able to lower knot probabilities in DNA far below equilibrium values. Some of the mechanisms to explain this behaviour rely on the localized nature of the knot $[15,16,17]$. In this context, it has been pointed out that supercoiling could be an important ingredient in localising polymer entanglement [54]. Moreover, supercoiling was recently found to favor DNA unknotting [55]. It would therefore be interesting to apply the techniques presented in this paper to a model of supercoiled DNA and see how this new ingredient influences the distribution of knot sizes.

Finally, in a recent experiment, a knot was tied in DNA using optical tweezers, and its subsequent diffusion in the presence of a stretching force was monitored [56]. This work has been the inspiration for several theoretical studies $[49,57,58,59]$. In [59], it was predicted that due to the interplay of bending energy and confinement entropy the knot will reach a metastable, compact configuration in which it will diffuse, without spreading, until it reaches one of the chain ends. It remains an interesting task for future work to investigate this prediction with the methods presented in this paper.

\section{Acknowledgments}

This work was supported by FIRB01, MIUR-PRIN05 and the FWO-Vlaanderen.

\section{References}

[1] Liu L F, Depew R E and Wang J C 1976 Knotted single-stranded DNA rings: a novel topological isomer of circular single-stranded DNA formed by treatment with Escherichia coli omega protein. J. Mol. Biol. 106 439-52

[2] Liu L F, Perkocha L, Calendar R and Wang J C 1981 Knotted DNA from bacteriophage capsids Proc. Natl. Acad. Sci. 78 5498-502

[3] Arsuaga J, Vazquez J, Trigueros S, Sumners D W and Roca J 2002 Knotting probability of DNA molecules confined in restricted volumes: DNA knotting in phage capsids Proc. Natl. Acad. Sci. 99 5373-7

[4] Shaw S Y and Wang J C 1993 Knotting of a DNA chain during ring closure Science 260 533-6

[5] Rybenkov V V, Cozzarelli N R and Vologodskii A V 1993 Probability of DNA knotting and the effective diameter of the DNA double helix Proc. Natl. Acad. Sci. 90 5307-11

[6] Delbruck M 1962 Knotting problems in biology Proc. Symp. Appl. Math 14 55-63

[7] Sumners D W and Whittington S G 1988 Knots in self-avoiding walks J. Phys. A: Math. Gen. 21 1689-94 
[8] Pippenger N 1989 Knots in random walks Disc. Appl. Math. 25 273-8

[9] Tesi M C, Janse van Rensburg E J, Orlandini E, Sumners D W and Whittington S G 1994 Knotting and supercoiling in circular DNA: A model incorporating the effect of added salt Phys Rev E 49 868-72

[10] Bates A D and Maxwell A 2005 DNA Topology (Oxford: Oxford University Press)

[11] Koster D A, Croquette V, Dekker C, Shuman S and Dekker N H 2005 Friction and torque govern the relaxation of DNA supercoils by eukaryotic topoisomerase IB Nature 434 671-4

[12] Rybenkov V V, Ullsperger C, Vologodskii A V and Cozzarelli N R 1997 Simplification of DNA topology below equilibrium values by type II topoisomerases Science $\mathbf{2 7 7} 690-3$

[13] Bates A D and Maxwell A 2007 Energy Coupling in Type II Topoisomerases: Why Do They Hydrolyze ATP? Biochemistry 46 7929-41

[14] Orlandini E and Whittington S G 2007 Statistical topology of closed curves: Some applications in polymer physics Rev. Mod. Phys. 79 611-42

[15] Vologodskii A V, Zhang W, Rybenkov V V, Podtelezhnikov A A, Subramanian D, Griffith J D and Cozzarelli N R 2001 Mechanism of topology simplification by type II DNA topoisomerases. Proc. Natl. Acad. Sci. 98 3045-9

[16] Buck G R and Zechiedrich E L 2004 DNA disentangling by type-2 topoisomerases J. Mol. Biol. 340 933-9

[17] Liu Z, Mann J K, Zechiedrich E L and Chan H S 2006 Topological information embodied in local juxtaposition geometry provides a statistical mechanical basis for unknotting by type-2 DNA topoisomerases J. Mol. Biol. 361 268-85

[18] Vologodskii A V, Crisona N J, Laurie B, Pieranski P, Katrich V, Dubochet J and Stasiak A 1998 Sedimentation and electrophoretic migration of DNA knots and catenanes J. Mol. Biol. 278 1-3

[19] Vanderzande C 1998 Lattice models of polymers (Cambridge: Cambridge University Press)

[20] Adams C C 2004 The knot book (Providence: American Mathematical Society)

[21] Guida R and Zinn-Justin J 1998 Critical exponents of the N-vector model J. Phys A: Math. Gen. 31, 8103-21

[22] Li B, Madras N and Sokal A D 1995 Critical exponents, hyperscaling, and universal amplitude ratios for two- and three-dimensional self-avoiding walks J. Stat. Phys. 80, 661-754

[23] Rolfsen D 1976 Knots and links (Wimington: Publish or Perish Press)

[24] Orlandini E, Tesi M C, Janse van Rensburg E J and Whittington S G 1996 Entropic exponents of lattice polygons with specified knot type J. Phys. A: Math. Gen. 29 L299-L303; Orlandini E, Tesi M C, Janse van Rensburg E J and Whittington S G 1998 Asymptotics of knotted lattice polygons, J. Phys. A: Math. Gen. 31 5953-67

[25] Katrich V, Olson W K, Vologodskii A, Dubochet J and Stasiak A 2000 Tightness of random knotting Phys Rev E 61 5545-9

[26] Guitter E and Orlandini E 1999 Monte Carlo results for projected self-avoiding polygons: A twodimensional model for knotted polymers J. Phys. A: Math. Gen. 32 1359-85

[27] Metzler R, Hanke A, Dommersnes P G, Kantor Y and Kardar M 2002 Equilibrium shapes of flat knots Phys. Rev. Lett. 88188101

[28] Maier B and Rädler J O 1999 Conformation and Self-Diffusion of Single DNA Molecules Confined to Two Dimensions Phys. Rev. Lett. 82 1911-4

[29] Duplantier B 1986 Polymer Network of fixed topology: renormalization, exact critical exponent $\gamma$ in two dimensions, and d=4- $\varepsilon$ Phys. Rev. Lett. 57 941-4

[30] Duplantier B 1989 Statistical mechanics of polymer networks of any topology J. Stat. Phys. 54 581-680

[31] Marcone B, Orlandini E, Stella A L and Zonta F 2005 What is the length of a knot in a polymer? $J$ Phys A: Math. Gen. 38 L15-L21

[32] Marcone B, Orlandini E, Stella A L and Zonta F 2007 Size of knots in ring polymers Phys Rev E 75 041105-11

[33] Orlandini E, Stella A L and Vanderzande C 2003 Polymer $\theta$-point as a knot delocalization transition 
Phys Rev E 68 031804-8

[34] Hanke A, Metzler R, Dommersnes P G, Kantor Y and Kardar M 2003 Tight and loose shapes in flat entangled dense polymers Eur. Phys. J. E 12 347-54

[35] Orlandini E, Stella A L and Vanderzande C 2004 Loose, flat knots in collapsed polymers J. Stat. Phys. 115 681-700

[36] Duplantier B and Saleur H 1987 Exact tricritical exponents for polymers at the $\theta$-point in two dimensions Phys. Rev. Lett. 59 539-42

[37] Virnau P, Kantor Y and Kardar M 2005 Knots in globule and coil phases of a model polyethylene J. Am. Chem. Soc. 127 15102-6

[38] De'Bell K, Lookman T 1993 Surface phase transitions in polymer systems Rev. Mod. Phys. 65 $87-113$

[39] Marcone B, Orlandini E and Stella A L 2007 Knot localization in adsorbing polymer rings Phys. Rev. E 76 051804-10

[40] Hegger R and Grassberger P 1994 Chain polymers near an adsorbing surface J Phys A: Math. Gen. 27 4069-81

[41] Ercolini E, Valle F, Adamcik J, Witz G, Metzler R, De Los Rios P, Roca J and Dietler G 2007 Fractal Dimension and Localization of DNA Knots Phys. Rev. Lett. 98058102

[42] Lai P Y 2002 Dynamics of polymer knots at equilibrium Phys. Rev. E 66 021805-12

[43] Trigueros S, Roca J, 2007, Production of highly knotted DNA by means of cosmid circularization inside phage capsids, BMC Biotechnology 7, 94.

[44] Orlandini E, Stella A L, Vanderzande C and Zonta F 2008 Slow topological time scale of knotted polymers J Phys A: Math. Gen. 41122002 (7pp)

[45] Rouse P E 1953 A theory of the linear viscoelastic properties of dilute. solutions of coiling polymers J. Chem. Phys. 21 1272-80

[46] de Gennes P-G 1979 Scaling concepts in polymer physics (Ithaca, NY: Cornell University Press)

[47] Shusterman R, Alon R S, Gavrinyov T and Krichevsky O 2004 Monomer Dynamics in Doubleand Single-Stranded DNA Polymers Phys. Rev. Lett. 92048303 (4pp)

[48] Downey J P, Crabb C C and Kovac J 1986 Dynamics of a face-centered cubic lattice model for polymer chains Macromolecules 19 2202-6

[49] Metzler R, Reisner W, Riehn R, Austin R, Tegenfeldt J and Sokolov I M 2006 Diffusion mechanisms of localised knots along a polymer Europhys. Lett. 76 696-702

[50] Stasiak A, Katrich V, Bednar J, Michoud D and Dubochet J 1996 Electrophoretic mobility of DNA knots Nature $\mathbf{3 8 4} 122$

[51] Rawdon E J, Dobay A, Kern J, Millett K, Piatek M, Plunkett P and Stasiak A 2008 Scaling behavior and equilibrium lengths of knotted polymers Macromolecules 41 4444-51

[52] Obukhov S P, Rubinstein M and Duke T 1994 Dynamics of a Ring Polymer in a Gel Phys. Rev. Lett. 73 1263-6

[53] Orlandini E, Stella A L and Vanderzande C, work in progress

[54] Marko J 1999 Coupling of intramolecular and intermolecular linkage complexity of two DNAs, Phys. Rev. E 59 900-12

[55] Burnier Y, Dorier J and Stasiak A 2008 DNA supercoiling inhibits DNA knotting, Nucl. Ac. Res. 36 4956-63

[56] Bao X R, Lee H J and Quake S R 2003 Behavior of complex knots in single DNA molecules Phys. Rev. Lett. 91 265506-4

[57] Huang L and Makarov D E 2007, Langevin dynamics simulations of the diffusion of molecular knots in tensioned polymer chains J. Phys. Chem. A 111 10338-44

[58] Vologodskii A 2006 Brownian Dynamics Simulation of Knot Diffusion along a Stretched DNA Molecule Biophys. J. 90 1594-97

[59] Grosberg A Y and Rabin Y, 2007 Metastable tight knots in a wormlike polymer, Phys. Rev. Lett. 99, 217801 